\title{
Labview Based ECG Patient Monitoring System for Cardiovascular Patient Using SMTP Technology
}

\author{
Om Prakash Singh, ${ }^{1}$ Dawit Mekonnen, ${ }^{1}$ and M. B. Malarvilii ${ }^{2}$ \\ ${ }^{1}$ Biomedical Engineering, JiT, Jimma University, 378 Jimma, Ethiopia \\ ${ }^{2}$ Department of Biotechnology and Medical Engineering, Faculty of Bioscience and Medical Engineering, Universiti Teknologi Malaysia, \\ 81310 Skudai, Johor, Malaysia
}

Correspondence should be addressed to Om Prakash Singh; bioom85@yahoo.com

Received 11 July 2015; Accepted 15 September 2015

Academic Editor: Norio Iriguchi

Copyright (C) 2015 Om Prakash Singh et al. This is an open access article distributed under the Creative Commons Attribution License, which permits unrestricted use, distribution, and reproduction in any medium, provided the original work is properly cited.

\begin{abstract}
This paper leads to developing a Labview based ECG patient monitoring system for cardiovascular patient using Simple Mail Transfer Protocol technology. The designed device has been divided into three parts. First part is ECG amplifier circuit, built using instrumentation amplifier (AD620) followed by signal conditioning circuit with the operation amplifier (lm741). Secondly, the DAQ card is used to convert the analog signal into digital form for the further process. Furthermore, the data has been processed in Labview where the digital filter techniques have been implemented to remove the noise from the acquired signal. After processing, the algorithm was developed to calculate the heart rate and to analyze the arrhythmia condition. Finally, SMTP technology has been added in our work to make device more communicative and much more cost-effective solution in telemedicine technology which has been key-problem to realize the telediagnosis and monitoring of ECG signals. The technology also can be easily implemented over already existing Internet.
\end{abstract}

\section{Introduction}

ECG is used to measure the rate and regularity of heartbeats, the presence of any damage to the heart, and the effects of drugs or devices used to regulate the heart (such as a pacemaker). Normally, the frequency range of ECG signal is $0.05-100 \mathrm{~Hz}$ and its dynamic range is $1-10 \mathrm{mV}$. The ECG signal as depicted in Figure 1 is characterized by five peaks and valleys labeled by the letters $P, Q, R, S$, and T. The performance of ECG analyzing system depends mainly on the accurate and reliable detection of the QRS complex, as well as T- and $\mathrm{P}$-waves. The $\mathrm{P}$-wave represents the activation of the upper chambers of the heart, the atria, while the QRS complex and $\mathrm{T}$-wave represent the excitation of the ventricles or the lower chamber of the heart. The detection of the QRS complex is the most important task in automatic ECG signal analysis. Once the QRS complex has been identified a more detailed examination of ECG signal including the heart rate and the ST segment can be performed $[1,2]$.
Most of the modern 12-lead ECG monitoring systems are based on Einthoven's triangle, Wilson central terminal, and Goldberger augmented leads [3].

The developed ECG device is implemented on the principle of Einthoven's triangle and used lead-II configuration, as it is known as a monitoring lead, given in Figure 2.

Based on above facts, there have been numerous attempts to develop medical systems similar to the work. Such efforts are primarily led by the academia but extending deeply into the industries. However, most research efforts have been focusing on either the vital sign monitoring aspect or the ECG feature extraction using standard databases both falling short of expectation. Having analyzed the existing solutions, this work vows to bridge the two major research efforts and bring out a more realizable product to directly benefit the consumers in the medical field.

This research work offers the following contributions to the produced system; foremost is the portable ECG monitoring platform based on a 3-lead system and a design 


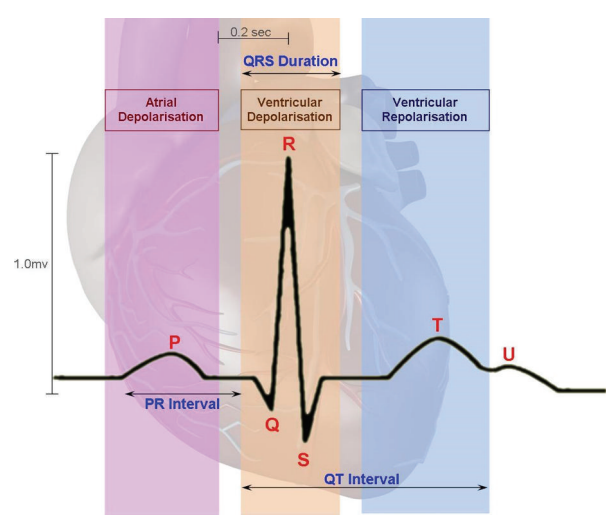

Figure 1: Peaks of ECG.

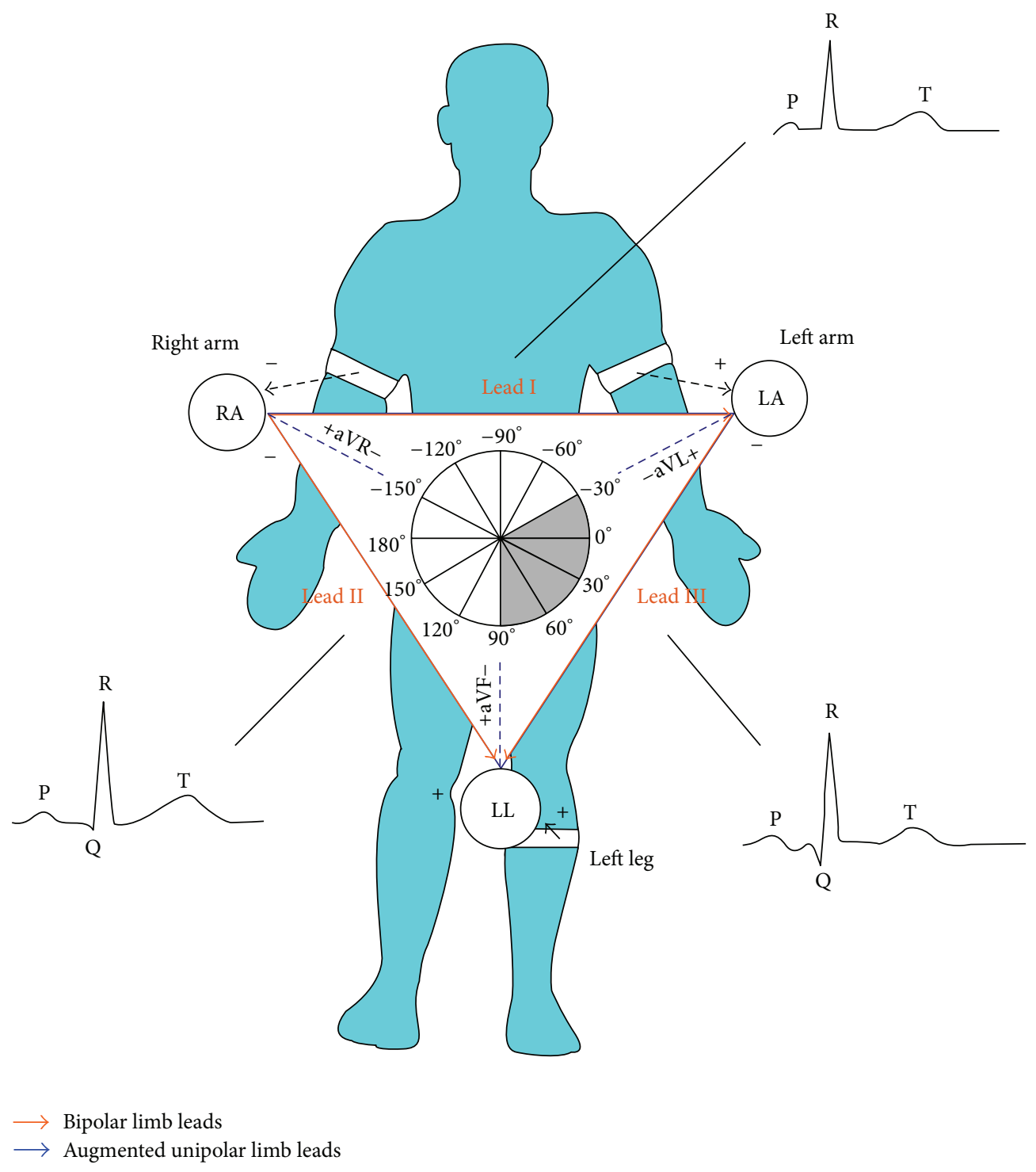

FIgURE 2: Electrode placement using a 3-wire cable. 


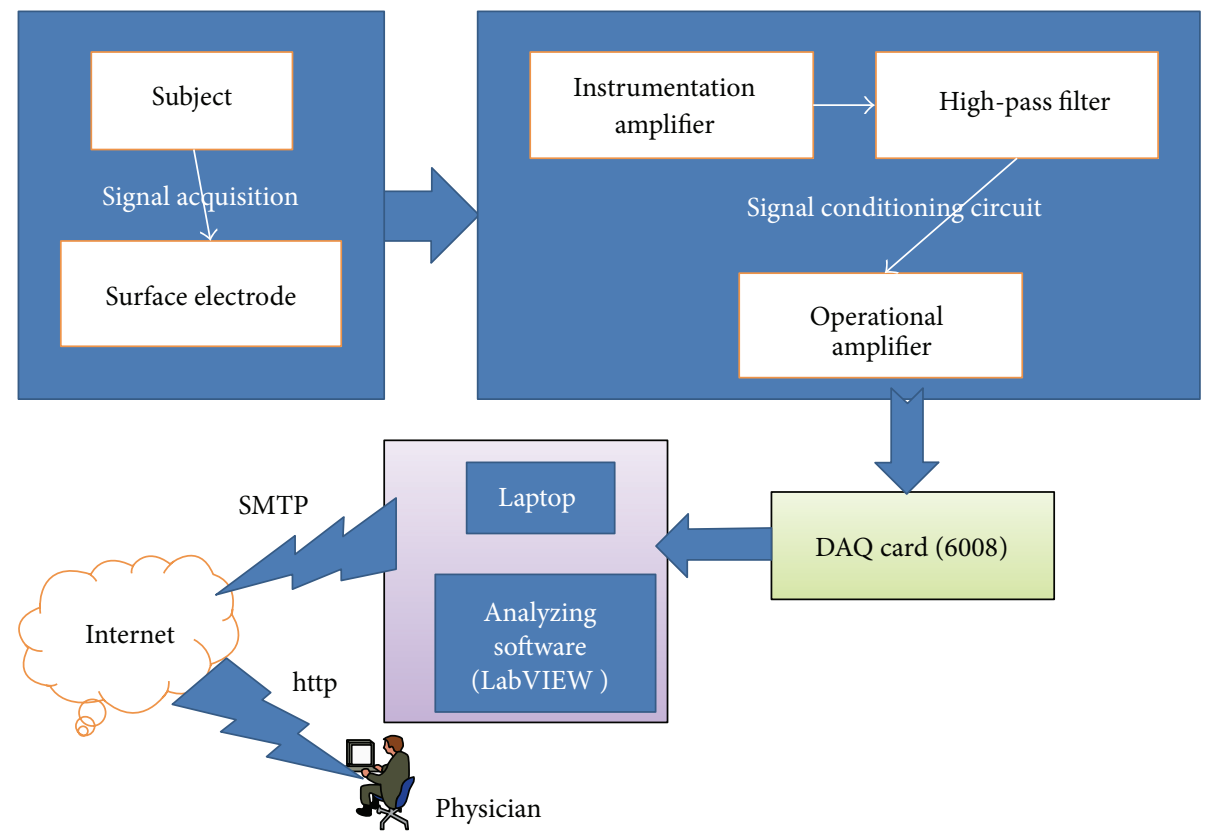

FIGURE 3: Block diagram of proposed ECG device.

under the NI DAQ card (6008). The ECG data was collected through the DAQ card to the PC/laptop and then transmitted to the end user (physician) through SMTP to analyze the patient condition.

\section{Material and Method}

The block diagram of the complete system is given in Figure 3. The complete design was divided into two parts: hardware and software.

The hardware part comprises instrumentation amplifier (AD620), some passive components, operational amplifier (LM741), DAQ card, and laptop whereas Labview is used as software. The software is used to exchange the data from analog to digital form, to perform the calculations, and to produce the ECG waveform onto the monitor. Each of the components in this block diagram is explained in detail in the following subsections [4].

2.1. Surface Electrode. The principle of the electrodes is to convert a physical parameter into an electrical output. The function of the transducer is to convert biological information into a quantifiable electrical signal. The transducer interface is provided using an electrode-electrolyte interface. The most preferable electrode is $\mathrm{Ag} / \mathrm{AgCl}$, as it reduces the impedance while using it and the gel is used for the proper contact in between the surface of the skin and electrode.

2.2. Signal Conditioning Circuit. After receiving raw ECG signal from the subject through electrode, it has to be processed in order to bring the signal in visible form and to limit the bandwidth of the signal. To do so, the instrumentation amplifier was used to amplify the tiny signal whereas passive and active components are used to design filter and to amplify it.

Figure 4 illustrates the constructive circuit diagram of signal conditioning. The circuit has been designed and tested in multisims to get the appropriate output of signal.

2.2.1. Instrumentation Amplifier. The voltage gain of the instrumentation amplifier is calculated using the following equation:

$$
\begin{aligned}
& G=1+\frac{49.4 \mathrm{k} \Omega}{R g}, \\
& G=1+\frac{49.4 \mathrm{k} \Omega}{1 \mathrm{k} \Omega}=1+49.4=50.4 .
\end{aligned}
$$

2.2.2. Operational Amplifier. The voltage gain of the operational amplifier is estimated using given formula as the used one is noninverting amplifier:

$$
\begin{aligned}
& G=1+\frac{R 3}{R 2}, \\
& G=1+\frac{200 \mathrm{k} \Omega}{1 \mathrm{k} \Omega}=1+200=201 .
\end{aligned}
$$




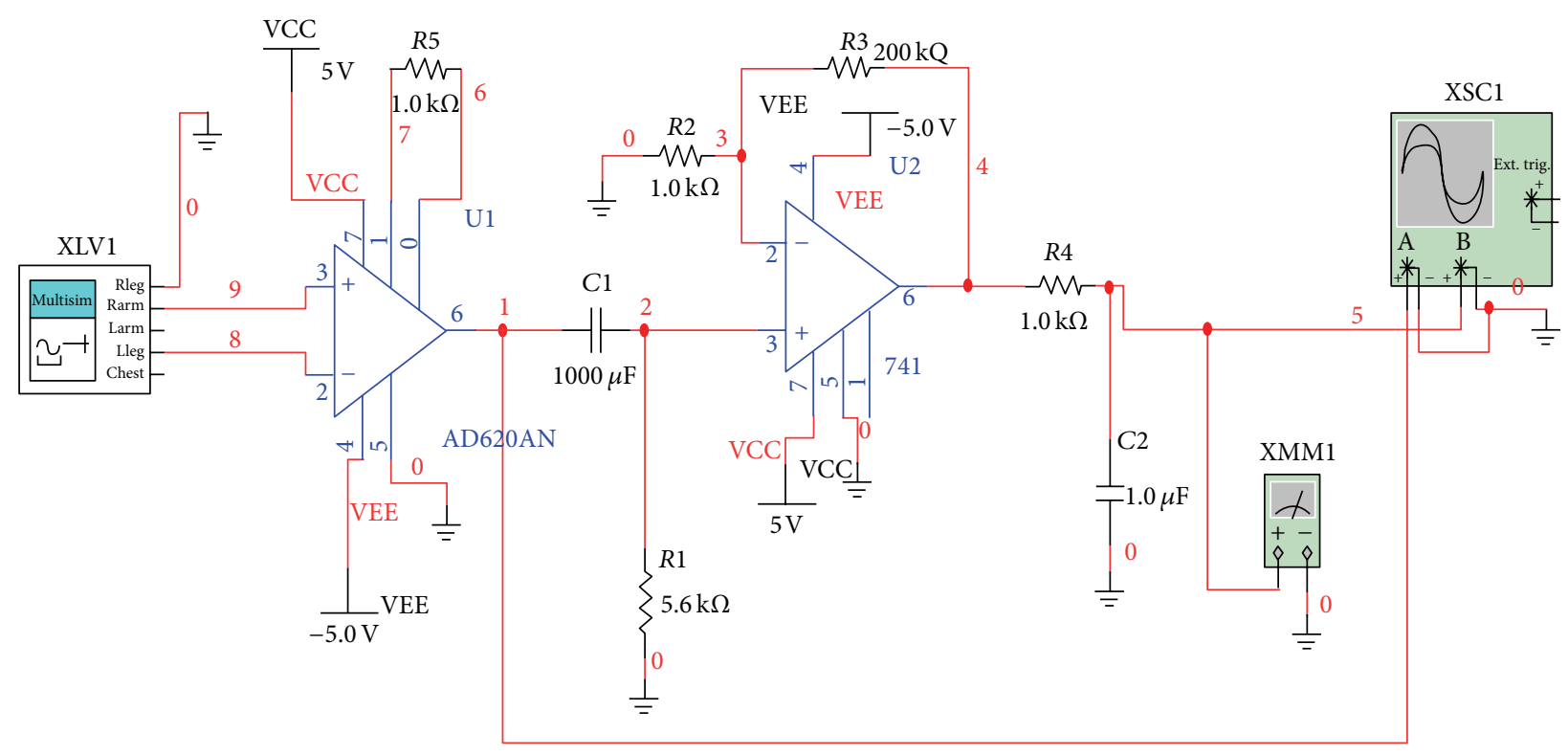

FIGURE 4: ECG signal acquisition and condition circuit.

2.2.3. High-Pass Filter. The output of the instrumentation amplifier is fed into the passive AC coupling with a cutoff frequency, as in (3), of $0.02 \mathrm{~Hz}$ such that high-pass filters

$$
\begin{aligned}
f_{\text {high-pass }} & =\frac{1}{2 \pi R 1 C 1} \\
& =\frac{1}{2 * 3.14 * 5.6 * 10^{3} * 1000 * 10^{-6}}, \\
f_{\text {high-pass }} & =\frac{5}{3.14 * 56}, \\
f_{\text {high-pass }} & =0.02 \mathrm{~Hz} .
\end{aligned}
$$

2.2.4. Low-Pass Filter. The second-stage amplified signal is fed into a low-pass filter with a cutoff frequency, as in (4), of $160 \mathrm{~Hz}$ for removing high frequency noise or movement artifacts:

$$
\begin{aligned}
f_{\text {low-pass }} & =\frac{1}{2 * \pi * R 4 * C 2} \\
& =\frac{1}{2 * 3.14 * 10 * 10^{3} * 1 * 10^{-6}}, \\
f_{\text {low-pass }} & =\frac{1000}{6.28}=159.2 \mathrm{~Hz} .
\end{aligned}
$$

2.3. DAQ Card (6008). The output of the signal conditioning circuit should be sent to the NI DAQ card for the conversion of signal from analog to digital as it has the inbuilt analog-todigital converter.

The given figure (Figure 5) gives the idea about the block diagram of NI USB-6008 which is a simple and low-cost multifunction I/O device from National Instruments [5]. It is used to digitize the amplified, filtered ECG signal. The NI USB-6008 card has 8 differential analog input channels and 12-bit analog-to-digital converter running at a sampling frequency up to $300 \mathrm{~Hz}$, which can be increased up to 200 $\mathrm{KHz}$. This satisfies the sampling requirements of the ECG signal.

\subsection{Laboratory Virtual Instrument Engineering Workbench} (Labview). It is a graphically programmed computer language for real-time instrumentation. It is a software package developed to build programs with symbols (icons) rather than writing out lines and lines of programming text. It uses symbols, terminology, and formats that are familiar to technicians, scientists, and engineers. Labview is programmed to act as an interface, helping pieces of hardware "communicate" with each other. Moreover, Labview offers built-in libraries that allow the user to work over the Internet and use different programming formats and systems.

2.5. Simple Mail Transfer Protocol (SMTP). Figure 6 shows the step of SMTP of how the data is processed throughout the different stages and transferred to client. It is a part of the application layer of the TCP/IP protocol. Using a process called "store and forward," SMTP moves your email on and across networks. It works closely with something called the Mail Transfer Agent (MTA) to send your communication to the right computer and e-mail inbox [http://support.aycontrol.com/].

\section{Designing Strategies}

The approach that has been followed in the designing of device is included in Figure 7. There were several stages like finding the appropriate electrodes to acquire the signal from body of the subject and analog signal conditioning circuit includes filtering and amplifying stage, DAQ card, and digital signal processing and display system. 


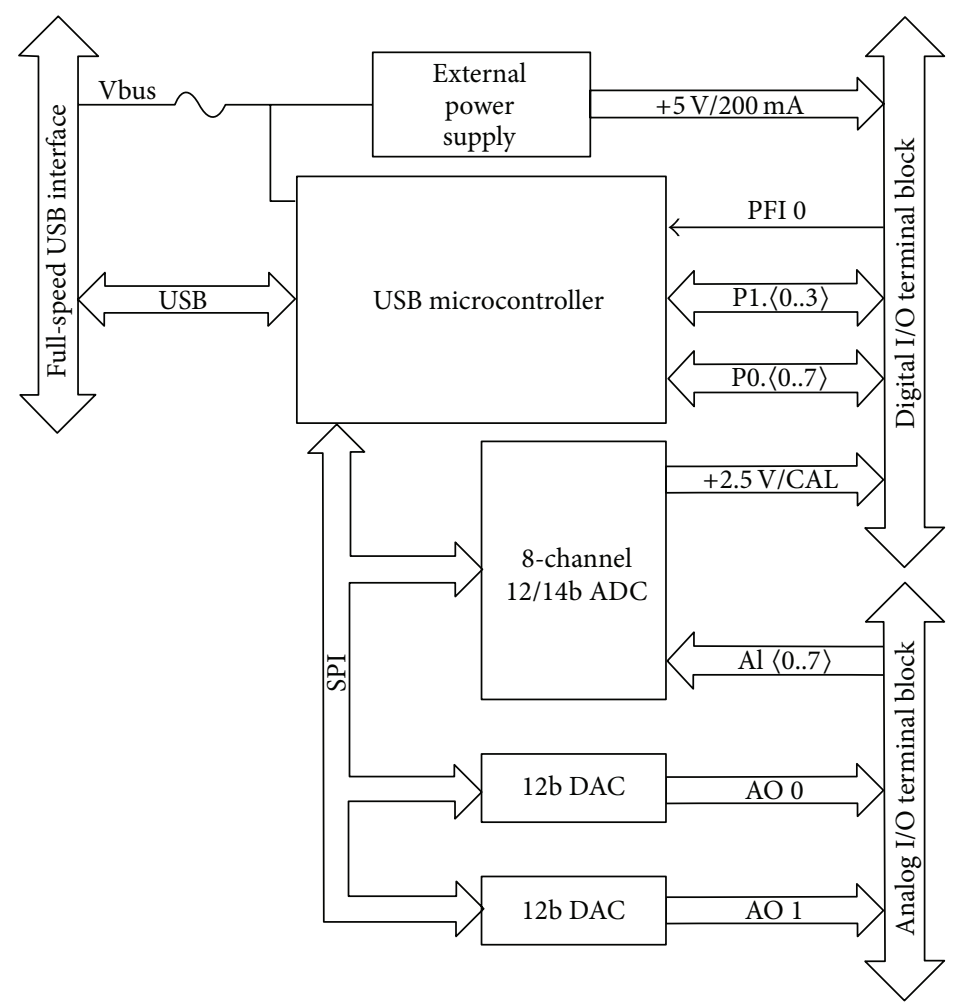

FIGURE 5: NI USB-6008 block diagram.

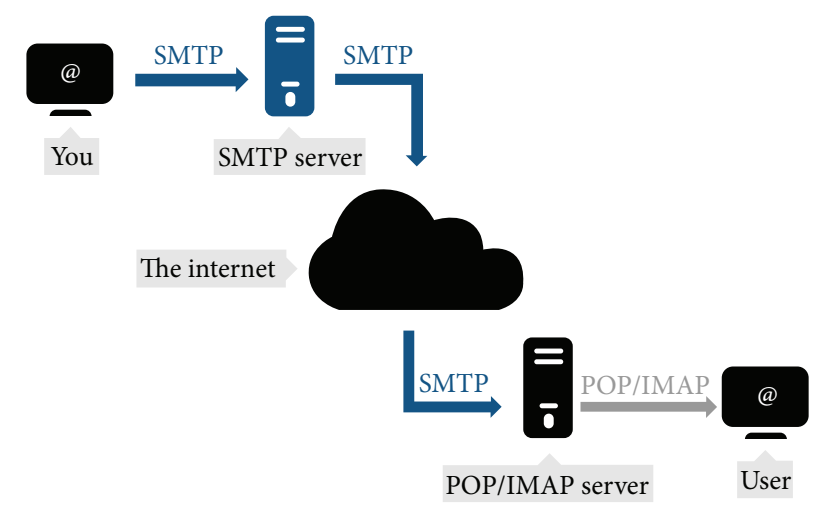

Figure 6: Simple Mail Transfer Protocol (refer to http://www.serversmtp.com/en/free-smtp-server).

\section{Designed Labview Interface}

The ECG system that has been developed is used for the continuous acquisition and chart recording of single input channels. It allowed user to record and save buffered analog ECG data from one or more individuals, which is continuously acquired into a circular buffer at the same time in that data previously retrieved from the buffer is plotted [68]. A common reason to read data while the acquisition is in progress is to process and display the data in virtual-real time.
4.1. Retrieving Input Signal. The analog signal is acquired from the bread board and converted to the digital one through DAQ. Digital signal processing is done accurately as needed in order to produce high signal-to-noise ratio so that the heart diagnostic system is precise.

4.2. Digital Signal Processing. Here, the digital filter is done to remove the power line interferences and other artifacts available in the signal. The necessary data processing to display real-time ECG had been performed.

4.3. Threshold and Peak Detector. The threshold and peak indicators are implemented after processing. The purpose of these indicators is to provide some feedback to the user (i.e., physician) regarding the patient's average heart rate.

Figure 8 gives the idea about the complete ECG system developed in Labview. The first part shows how the data have been acquired using data acquisition card (6008); immediate in next step, the retrieved data has been processed by Band Pass Filter as well as notch filter to remove the artifacts from the signal $[9,10]$. Furthermore, the algorithm is developed to detect the peak of the signal.

The following given equation is used to calculate the heart rate and to identify the arrhythmia condition $[11,12]$ :

$$
\text { Heart Rate }=\frac{60}{t_{2}-t_{1}},
$$

with $t_{1}$ being occurrence of first $\mathrm{R}$ wave and $t_{2}$ being occurrence of second $\mathrm{R}$ wave. 
LabVIEW based patient monitoring system for cardiovascular patient and EGG data transferred using SMTP technology

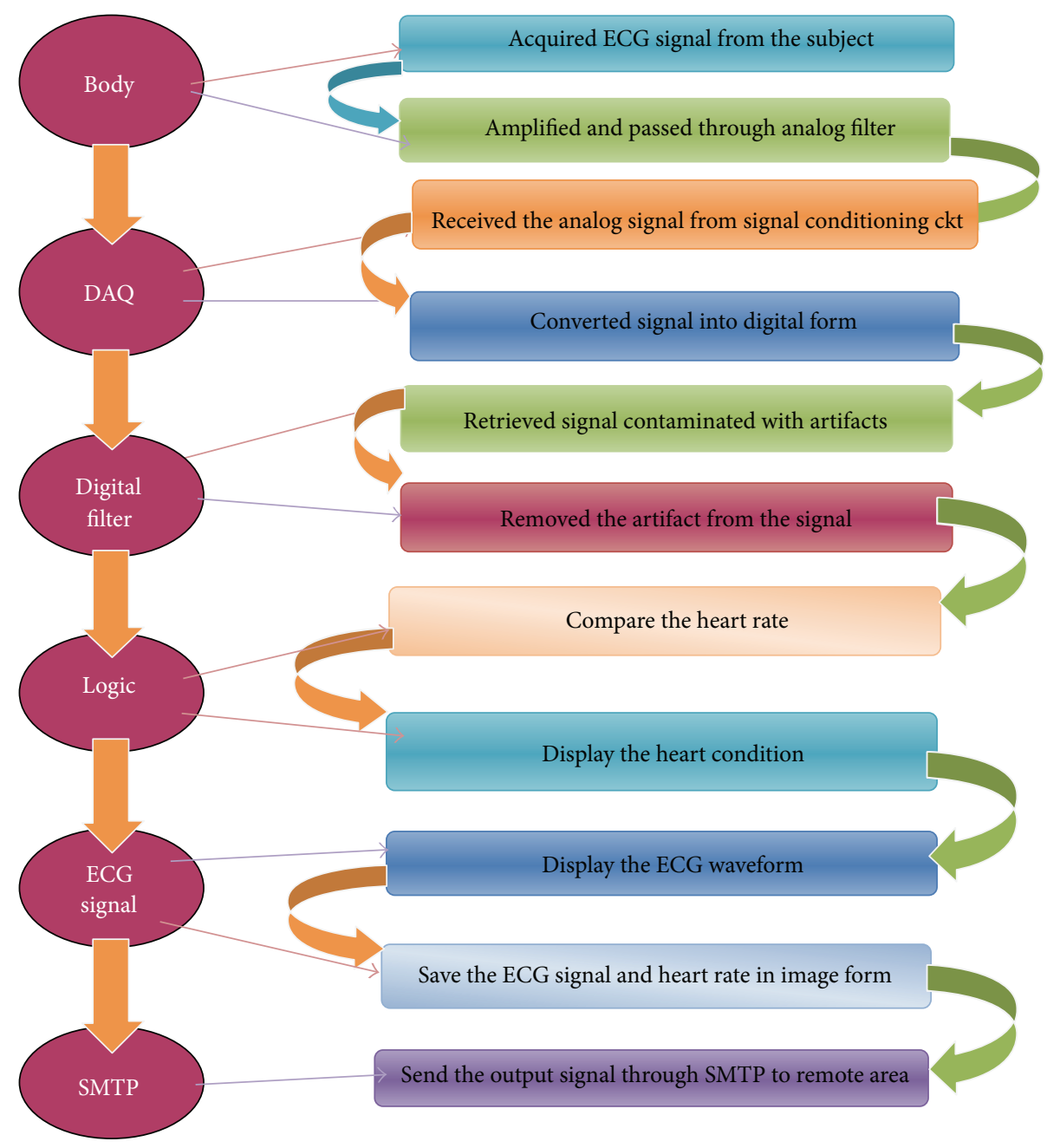

FIGURE 7: Designing strategy.

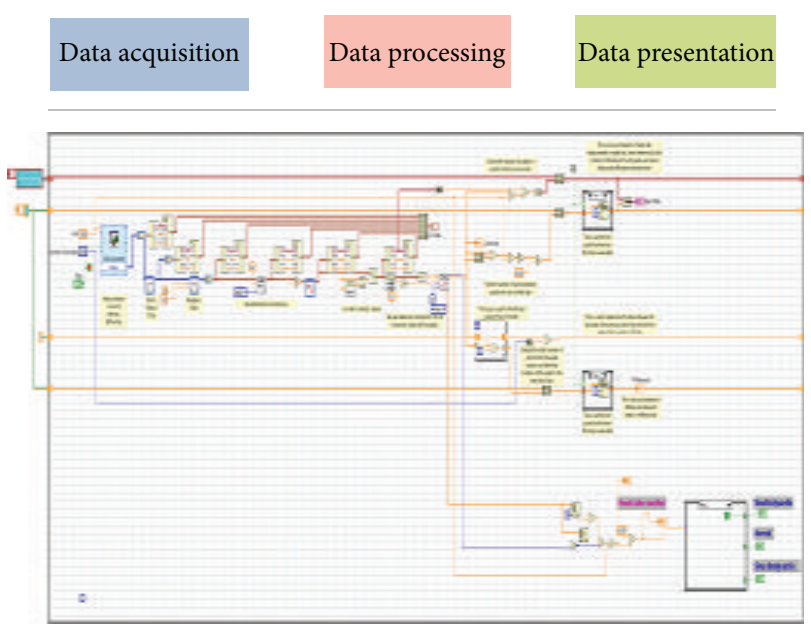

FIgURE 8: Acquired, processed, and calculated heart rate.
4.4. Display System Tool (Toshiba). After processing raw ECG signal, the signal has been displayed onto the laptop as shown in Figure 9. The display too is compatible with the prerequisite of the Labview software.

4.5. SMTP (Simple Mail Transfer Protocol). Finally, the developed SMTP tool is used to send image file of acquired ECG signal through Email Express VI, shown in Figure 10 that represents the front panel and Figure 11 that represents the block panel developed using Labview which allows sending the data quickly through e-mails from Labview to a health care centre or a physician.

\section{Result}

The given system is based on the principle of heart rate monitoring; it is able to produce the results shown in 


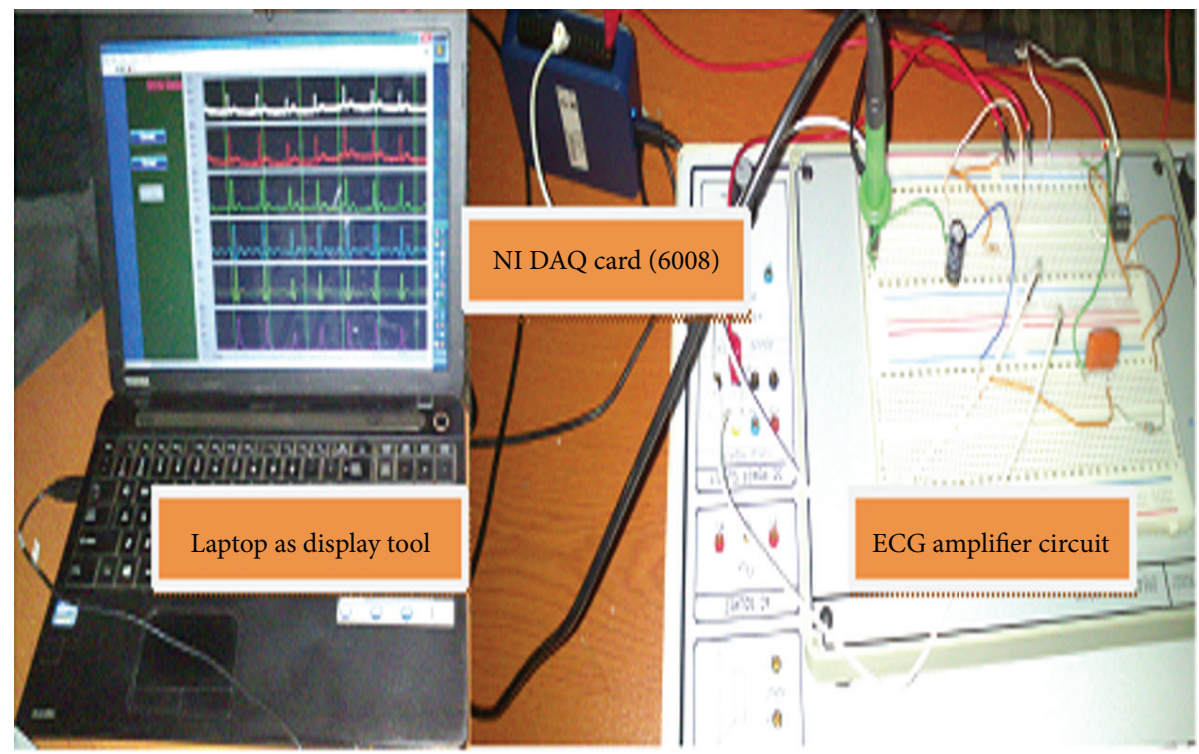

FIgUre 9: Proposed ECG device.

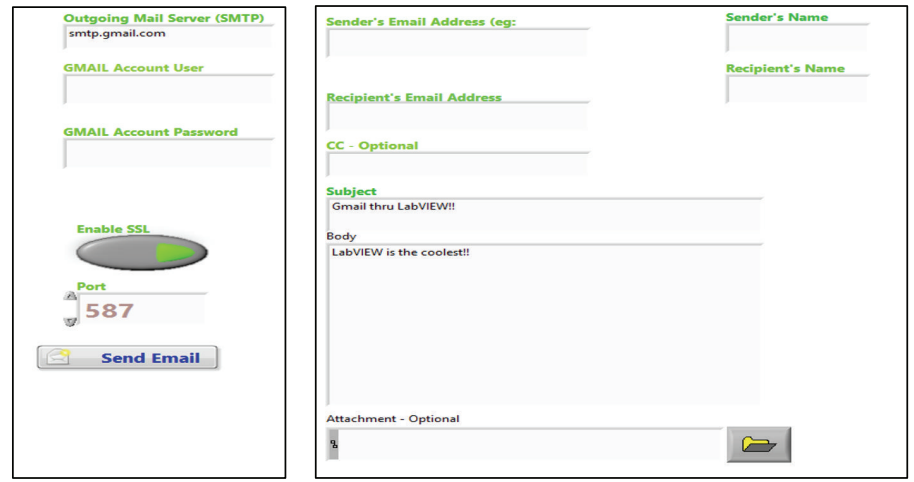

FIgURE 10: Front panel of SMTP.

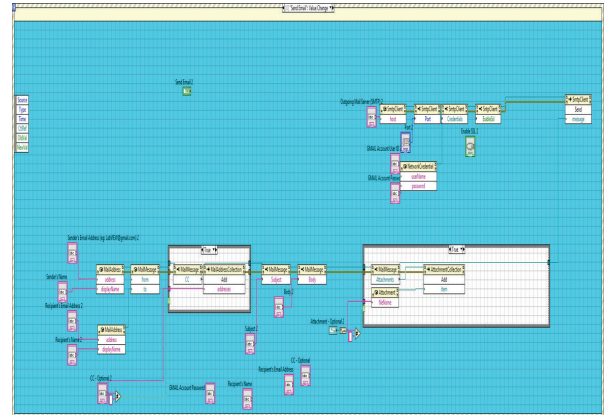

FIGURE 11: Block panel of SMTP.

Figure 12, which show accurate ECG signals and the correct calculation of both a resting and an elevated heart rate. Initially, there was an issue with the system regarding doctor notification subsystem which has been short by validating the proposed concept by running the VI at home, which sent both text and e-mail to our "doctor" notifying them that there was a detected problem with the patient's heart. It brings the freedom for the physician as well as for the doctor to check up on patients hearts from time to time by seeing real-time waveforms as shown in Figure 12.

The proposed device is functioning well once all the hardware connected properly to meet the criteria for the proposed idea. As a whole, it is very reliable and portable as well as cost effective.

\section{Discussion}

Most of the work has been done based either on hardware or on software. In the case of hardware, to transmit the ECG data, transmitter and receiver had been used, which increases the cost of the device, whereas the developed one integrated with hardware along with software to transmit the 


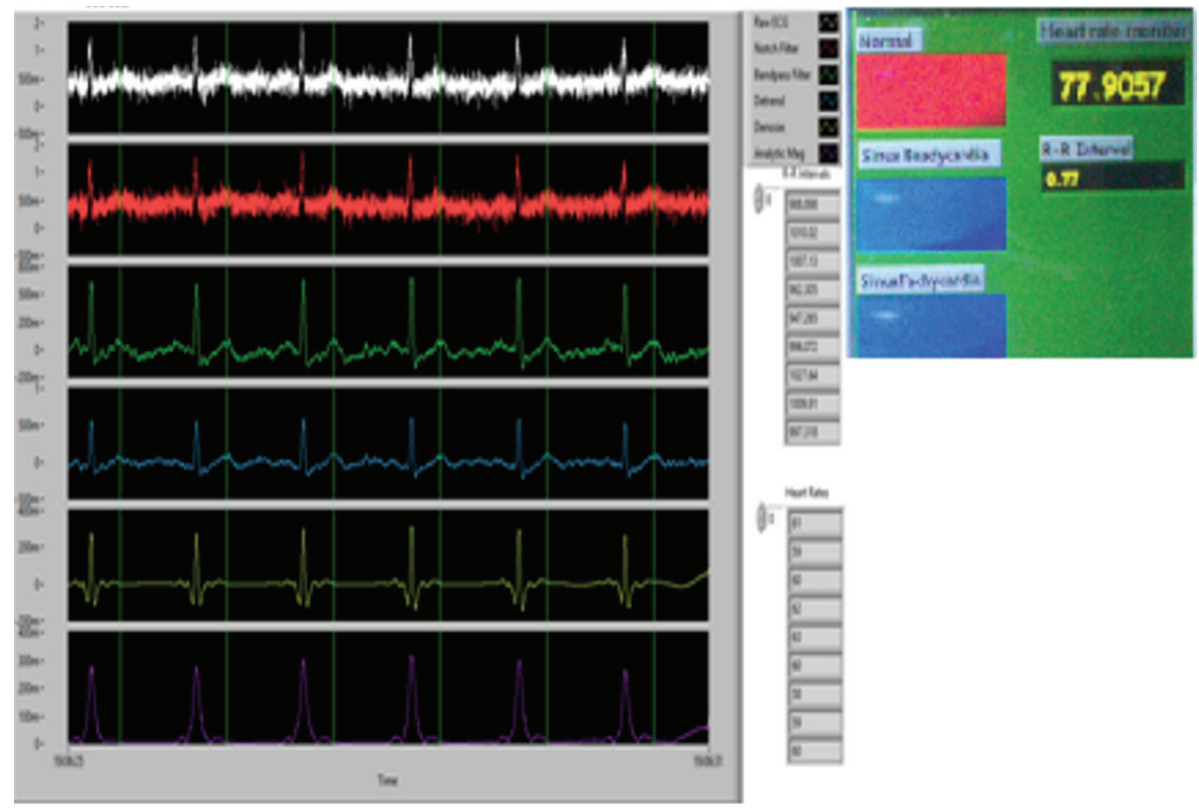

FIGURE 12: Acquired, processed ECG signal.

data anywhere using SMTP technology. Thus, the complete device becomes more user friendly as well as cost effective.

\section{Conclusion and Future Work}

In this paper, the low-cost biomedical measurement system with the ability of storage in digital format as well as sending the data to the remote area has been presented. The hardware implementations using commercially available devices and the software written in Labview program for continuously monitoring ECG data have been described. The proposed measurement system is also capable of sending the data through SMTP to the physician or health care centre with no time. The proposed system could be modified by increasing the number of channels.

\section{Conflict of Interests}

The authors declare that there is no conflict of interests regarding the publication of this paper.

\section{Acknowledgments}

One of the authors would like to express their gratitude to their colleagues for their help in accomplishing this work. This study is supported under a project funded by Universiti Teknologi Malaysia and by Ministry of Higher Education, Malaysia, under Grant no. R.J130000.7845.4F473.

\section{References}

[1] P. Khanja, S. Wattanasirichaigoon, J. Natwichai, L. Ramingwong, and S. Noimanee, "A WEB base system for ECG data transferred using ZIGBEE/IEEE technology," in Proceedings of the 3rd International Symposium on Biomedical Engineering (ISBME '08), Bangkok, Thailand, 2008.

[2] C. Saritha, V. Sukanya, and Y. Narasimha Murthy, "ECG signal analysis using wavelet transforms," Bulgarian Journal of Physics, vol. 35, Article ID 13100157, pp. 68-77, 2008.

[3] C. T. Weay, F. C. Horng, and S. Nadarajah, PC Based ECG Monitoring System, School of Computer Engineering, Nanyang Technological University, Singapore, http://www3.ntu.edu.sg/ eee/urop/congress2003/Proceedings/abstract/NTU_SCE/FohSCE.pdf.

[4] A. A. M. Adam and M. B. M. Amin, "Design and implementation of portable PC-based ECG machine," International Journal of Sciences: Basic and Applied Research, vol. 15, no. 2, 2014.

[5] User Guide and Specifications NI USB-6008/6009, http://ni .com/manuals.

[6] A. Wongjan, A. Julsereewong, and P. Julsereewong, "Continuous measurements of ECG and $\mathrm{SpO}_{2}$ for cardiology information system," in Proceedings of the International MultiConference of Engineers and Computer Scientists (IMECS '09), vol. 2, Hong Kong, March 2009.

[7] M. Lascu and D. Lascu, "LabVIEW based biomedical signal acquisition and processing," in Proceedings of the 7th WSEAS International Conference on Signal, Speech and Image Processing (SSIP '07), pp. 38-43, Athens, Greece, August 2007.

[8] H. Sahool and K. Biswal, "Patient monitoring system for cardiovascular patient with body temperature using lab VIEW," International Journal of Engineering Research and Development, vol. 6, no. 8, pp. 27-30, 2013.

[9] A. S. Khaing and Z. M. Naing, "Quantitative investigation of digital filters in electrocardiogram with simulated noises," International Journal of Information and Electronics Engineering, vol. 1, no. 3, 2011.

[10] P. Le-Huy, J. P. L-Huillier, Y. Omerzouk, and E. Yvroud, "Microprocessor-based ambulatory ecg monitoring system," in Proceedings of the 7th Annual Symposium on Computer Applications 
in Medical Care, pp. 917-918, IEEE, Washington, DC, USA, October 1983.

[11] M. K. Islam, A. N. M. M. Haque, G. Tangim, T. Ahammad, and M. R. H. Khondokar, "Study and analysis of ECG signal using MATLAB \& LABVIEW as effective tools," International Journal of Computer and Electrical Engineering, vol. 4, no. 3, pp. 404408, 2012.

[12] National Instruments, LabVIEW for ECG Signal Processing, 2012. 

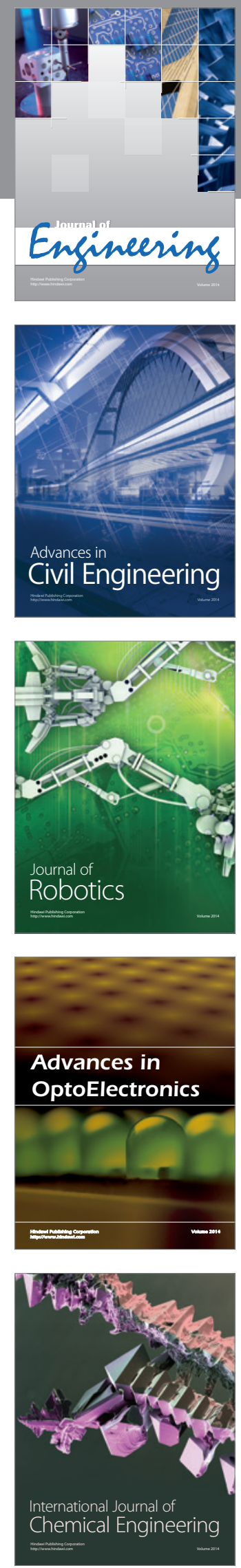

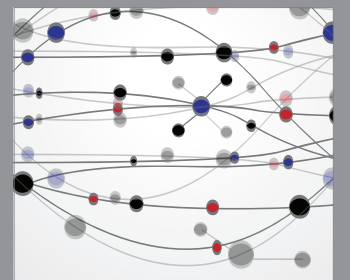

The Scientific World Journal
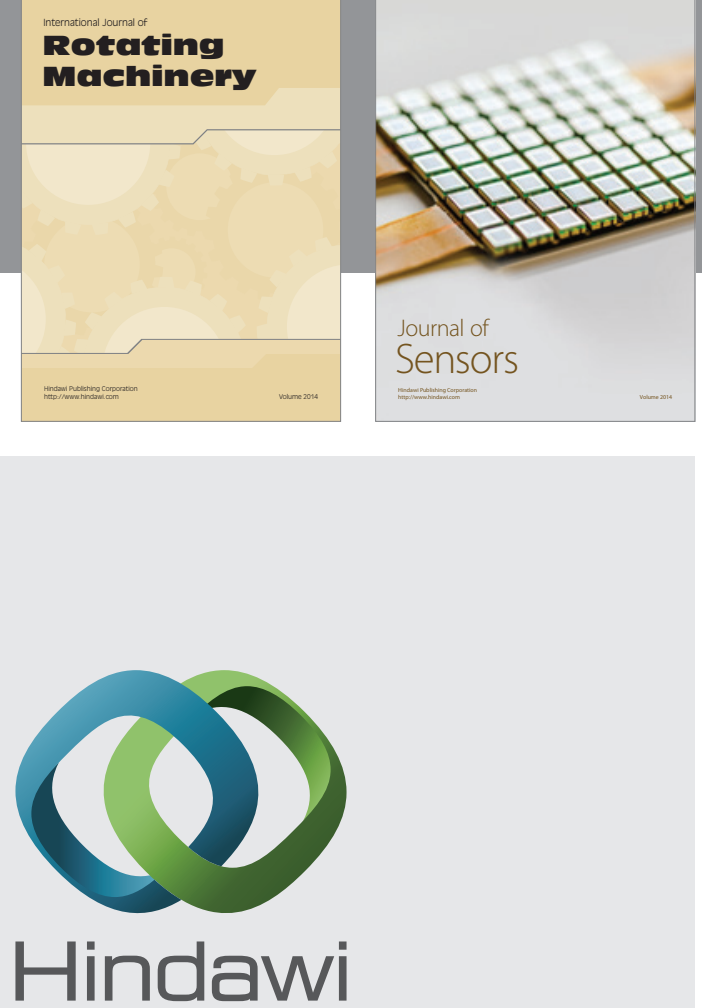

Submit your manuscripts at http://www.hindawi.com
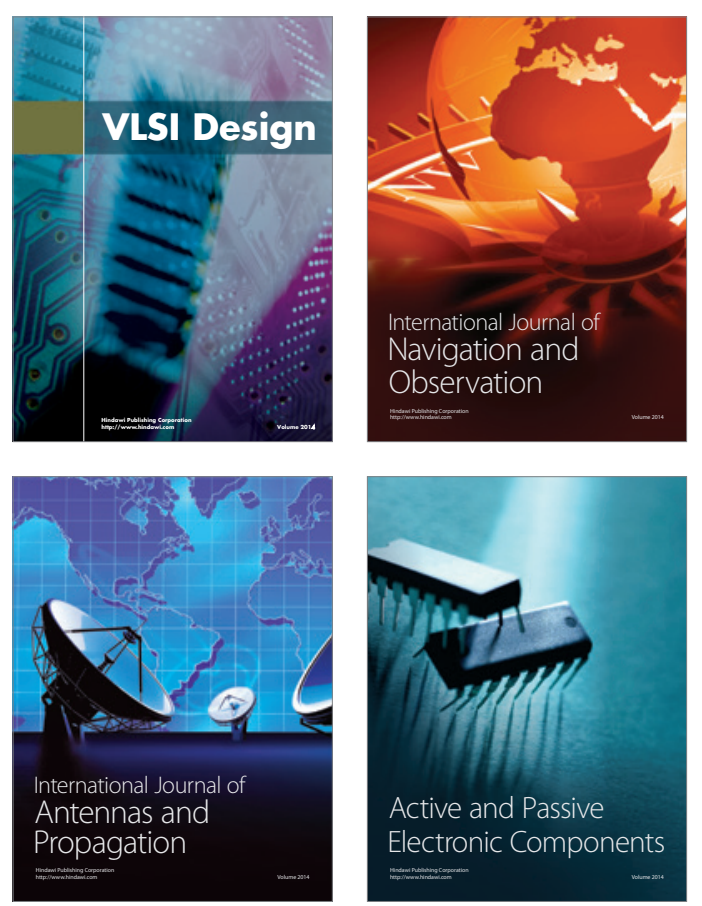
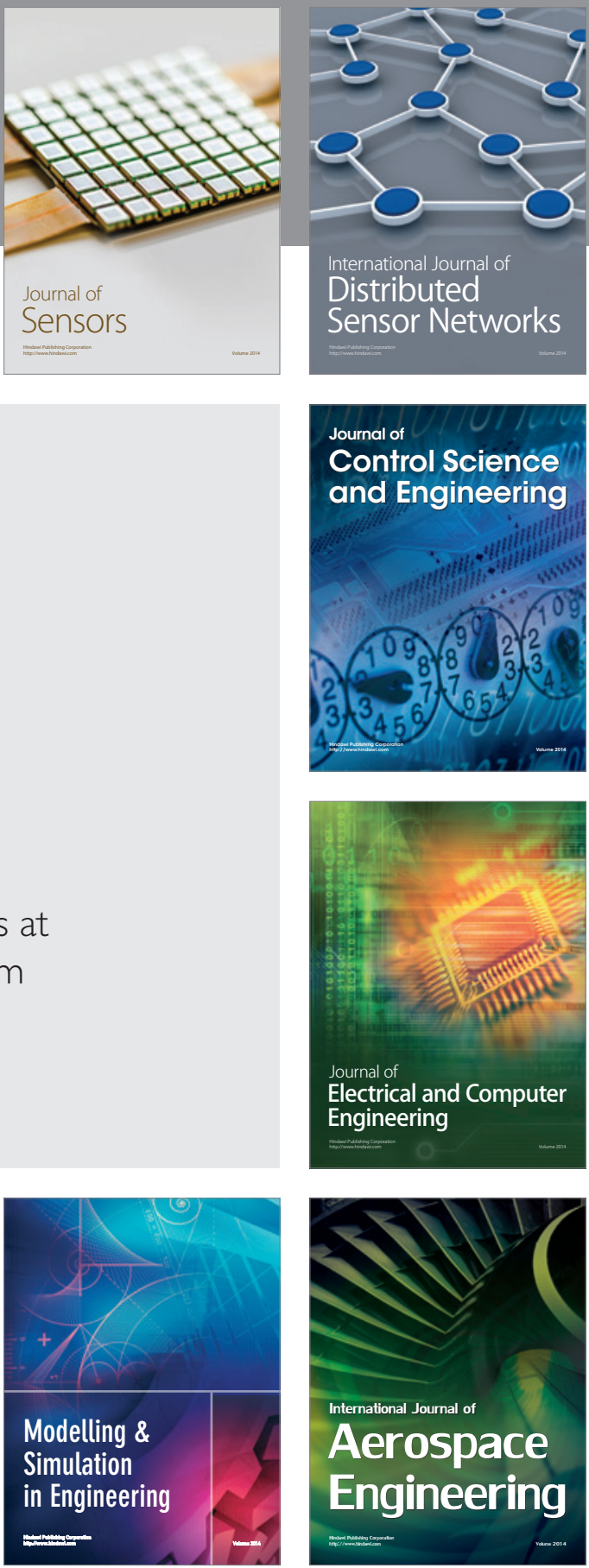

Journal of

Control Science

and Engineering
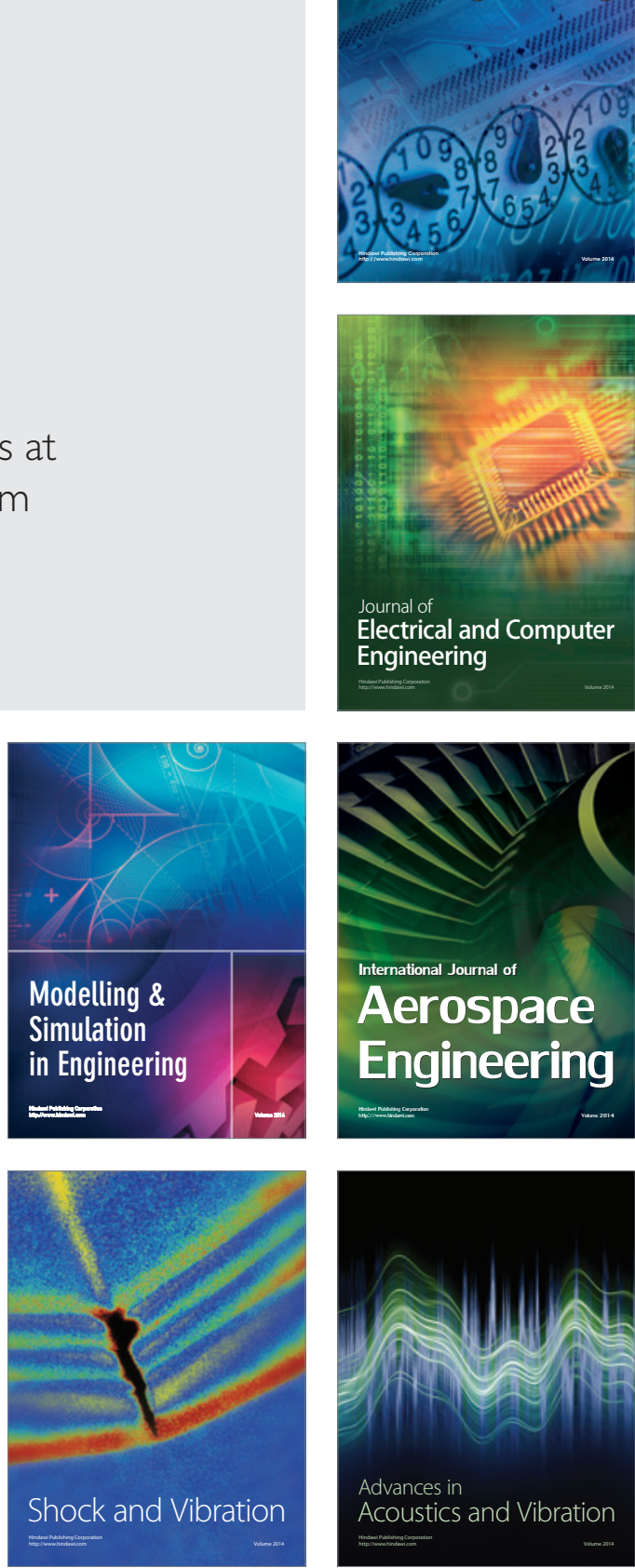